\title{
Human aging and somatic point mutations in mtDNA: A comparative study of generational differences (grandparents and grandchildren)
}

\author{
Anderson Nonato do Rosário Marinho ${ }^{1}$, Milene Raiol de Moraes ${ }^{1,2}$, Sidney Santos ${ }^{1}$ \\ and Ândrea Ribeiro-dos-Santos ${ }^{1}$ \\ ${ }^{1}$ Laboratório de Genética Humana e Médica, Instituto de Ciências Biológicas, \\ Universidade Federal do Pará, Belém, PA, Brazil. \\ ${ }^{2}$ Prefeitura Municipal de Belém, Belém, PA, Brazil.
}

\begin{abstract}
The accumulation of somatic mutations in mtDNA is correlated with aging. In this work, we sought to identify somatic mutations in the HVS-1 region (D-loop) of mtDNA that might be associated with aging. For this, we compared 31 grandmothers (mean age: $63 \pm 2.3$ years) and their 62 grandchildren (mean age: $15 \pm 4.1$ years), the offspring of their daughters. Direct DNA sequencing showed that mutations absent in the grandchildren were detected in a presumably homoplasmic state in three grandmothers and in a heteroplasmic state in an additional 13 grandmothers; no mutations were detected in the remaining 15 grandmothers. However, cloning followed by DNA sequencing in 12 grandmothers confirmed homoplasia in only one of the three mutations previously considered to be homoplasmic and did not confirm heteroplasmy in three out of nine grandmothers found to be heteroplasmic by direct sequencing. Thus, of 12 grandmothers in whom mtDNA was analyzed by cloning, eight were heteroplasmic for mutations not detected in their grandchildren. In this study, the use of genetically related subjects allowed us to demonstrate the occurrence of age-related (> 60 years old) mutations (homoplasia and heteroplasmy). It is possible that both of these situations (homoplasia and heteroplasmy) were a long-term consequence of mitochondrial oxidative phosphorylation that can lead to the accumulation of mtDNA mutations throughout life.
\end{abstract}

Key words: aging, mtDNA, mitochondrial somatic mutations.

Received: January 11, 2010; Accepted: July 26, 2010.

Mitochondrial DNA (mtDNA) is located in the mitochondrial matrix, a highly oxidative microenvironment where oxidative phosphorylation (OXPHOS) generates the ATP required for cellular energy. The presence of free radicals (ROS), together with an inefficient repair system, makes mtDNA vulnerable to oxidative damage (Richter et al., 1988; Rose et al., 2002; Khaidakov et al., 2003) and favors the generation of somatic mutations (He et al., 2010). In mammals, the occurrence of these mutations in mtDNA is correlated with aging (Harman, 1981; Ortopassi and Arnheim, 1990; Corral-Debrinski et al., 1992; Wallace, 1992; Miquel, 1998). Khaidakov et al. (2003) recently reported that mice 22 months old had a higher frequency of substitutions in the D-loop of the mtDNA control region than two-month-old mice; this frequency was $\sim 1000$ times greater than for nuclear genes. The authors estimated that at 22 months of age, which corresponds to three fourths of the normal life expectancy of laboratory mice, most mtDNA

Send correspondence to Ândrea Ribeiro-dos-Santos. Laboratório de Genética Humana e Médica, Instituto de Ciências Biológicas, Universidade Federal do Pará, Cidade Universitária Prof. José Silveira Netto, Avenida Augusto Corrêa 1, 66075-970 Belém, PA, Brazil. E-mail: akely@ufpa.br. molecules carried multiple point mutations, a situation possibly representative of other mammals.

In humans, mutations in the D-loop of mtDNA have been investigated in cultured fibroblasts from young and elderly normal individuals (Michikawa et al., 1999). Point mutations detected in elderly individuals were not present in youngsters. In particular, the transversion 16,414 $(\mathrm{T} \rightarrow \mathrm{G})$ was observed in $57 \%$ of fibroblasts from elderly subjects.

Parsons et al. (1997) compared the DNA sequences of two hypervariable segments in the control region of closely related maternal relatives, i.e., mother:child, grandmother:grandchild and sibling pairs, from 134 independent mtDNA lineages in 327 generational events. The ten substitutions observed resulted in an empirical rate of 1 in 33 generations (2.5/site/Myr), roughly 20 fold higher than estimates from phylogenetic studies. One of the processes that could account for this difference was the elimination of new substitutions by genetic drift or by selection over time. The authors also observed a tissue difference in substitution rates, i.e., wherase in blood samples the estimated rate was eight substitutions $/ 153$ generations $(0.052 /$ generation or 
4.3/site/Myr), in cell lines (DNA from CEPH pedigree cell lines) the estimated rate was two substitutions/174 generations $(0.011 /$ generation or $0.94 /$ site/Myr). This difference may possibly reflect enhanced selection in culture that would favor the original sequences.

In contrast to age, mental disease mental disease does not appear to affect the frequency of these mutations. For example, Chinnery et al. (2001) examined the frequency of mutations in the D-loop region of mtDNA in 113 frozen brain tissue samples from 31 normal elderly individuals with no clinical evidence of dementia before death (mean age: $71.7 \pm 10.2$ years), 35 individuals with Alzheimer's disease (mean age: $77.9 \pm 9.6$ years) and 47 individuals with dementia (mean age: $79.1 \pm 5.5$ years). In none of these three groups was there an accumulation of somatic point mutations in this mitochondrial genome region, indicating that neurodegenerative disease did not influence the frequency of mutations.

In this study, we compared the sequence of the mtDNA D-loop of healthy grandmothers and their daughters' children in order to examine the influence of age on the occurrence of somatic mutations. The use of genetically related subjects allowed us to differentiate between inherited and age-related mutations. The sample consisted of 31 unrelated grandmothers, (mean age: $63 \pm 2.3$ years), and their 62 grandchildren (mean age: $15 \pm 4.1$ years), the offspring of their daughters. Most of the families had one grandmother and two grandchildren. These families were from in the metropolitan area of the city of Belém, in the state of Pará, northern Brazil. General information about the individuals' health was extracted from a standard questionnaire. All of the participants or their legal guardians signed an informed consent form. The study was done in accordance with the Declaration of Helsinki (2000) issued by the World Medical Association.

Mutations were first screened in all 93 individuals by direct sequencing to allow grandmother $v s$. grandchild comparisons. Somatic mutations were subsequently validated by sequencing cloned DNA.

DNA was extracted by a phenol-chloroform method and precipitated with ethanol (Sambrook et al., 1989). Initially, the mtDNA D-loop of all 93 samples was amplified by PCR and sequenced. PCR amplification was done in a GeneAmp PCR system 9700 (Life Technologies, Foster City, CA, USA) with forward primer 5' CAC CAT TAG CAC CCA AAG CTA 3' and reverse primer 5' CCA GTT CCC TGG GGA TAG ACT 3', which amplifed a 422 bp fragment located in the coding region (D-loop). The reactions were done in a final volume of $25 \mu \mathrm{L}$, containing $100 \mathrm{ng}$ of DNA/ $\mu \mathrm{L}, 10 \mathrm{mM}$ Tris- $\mathrm{HCl}, \mathrm{pH} 8.5,50 \mathrm{mM} \mathrm{KCl}$, $1.5 \mu \mathrm{M} \mathrm{MgCl}_{2}, 1.25 \mathrm{mM}$ of each dNTP, $1.25 \mathrm{mM}$ of each primer and $1 \mathrm{U}$ of platinum Taq DNA polymerase. Thermal cycling consisted of an initial amplification cycle at $95^{\circ} \mathrm{C}$ for $11 \mathrm{~min}, 35$ cycles at $94{ }^{\circ} \mathrm{C}$ for $1 \mathrm{~min}, 50^{\circ} \mathrm{C}$ for $30 \mathrm{~s}$ and
$72{ }^{\circ} \mathrm{C}$ for $1 \mathrm{~min} 20 \mathrm{~s}$, and a final extension step at $72{ }^{\circ} \mathrm{C}$ for $45 \mathrm{~min}$. The final PCR product was purified using a CONCERT kit (Life Technologies, Carlsbad, CA, USA) and sequenced in an automated 377 ABI Prism apparatus with an ABI PRISM Dye Terminator Cycle sequencing kit (Life Technologies, Foster City, CA, USA).

Precautions taken to prevent reading errors in the DNA sequences included: (i) sequencing each sample at least twice, using forward and reverse primers that covered the same target region in both directions, (ii) alignment of the sequences using the Bioedit program (Ayres et al., 2007) and (iii) manual confirmation (by two independent observers) of the chromatogram sequences, individually and without resorting to the reading program. Thus, all mutations were confirmed four times: twice by forward and reverse sequencing, and twice by two observers who determined the sequences individually. This first phase of the study generated 186 sequences for the 93 individuals (Table S1).

DNA from 12 grandmothers (three homoplasmic samples and nine randomly chosen heteroplasmic samples) was cloned using a $\mathrm{pCR}^{\circledR} 2.1-\mathrm{TOPO}^{\circledR}$ vector from the TOPO TA cloning ${ }^{\circledR}$ kit (Life Technologies, Carlsbad, CA, USA). Ten clones from each individual (total of 120 samples) were sequenced, with two sequencing replicates for each clone (total of 240 sequences; Table S2). The plasmids with inserts were sequenced in an automated sequencer using an ABI3130 PRISM Dye Terminator Cycle sequencing kit (Life Technologies, Foster City, CA, USA).

In the 31 families investigated by direct sequencing (Table S1), three grandmothers from families 21, 27 and 30 (3/31 families, 9.7\%) had homoplasmic point mutations that were not observed in their grandchildren. Two mutations were $\mathrm{C} \rightarrow \mathrm{T}$ transitions at nucleotide positions 16,111 and 16,188; the third was a $\mathrm{T} \rightarrow \mathrm{C}$ transition at position 16,131. In another 13 grandmothers ( $42 \%$; families $1,3,5$, $6,7,8,9,11,13,15,19,25$ and 26), there was evidence of heteroplasmy (overlapping peaks in the electropherograms). In family 25 , in which five grandchildren were investigated (Table S1), there was a homoplasmic deletion at nucleotide position 16,384 in three grandchildren that was absent in the grandmother and in the other two grandchildren investigated.

Direct sequencing revealed 16 families with possible mutations between generations 1 and 3,12 of which were analyzed further by cloning and sequencing (families 1, 3, $5,8,9,11,13,15,19,21,27,30$; Table S2). The sequences obtained for grandmothers were aligned and compared with the reference sequence (Anderson et al., 1981, 1999) and then compared with those of their grandchildren obtained by direct sequencing. This procedure allowed distinction between inherited and non-inherited mutations, as well as the identification of mutations present in one generation but not in the other. The results can be summarized as follows: 
a) Nine grandmothers $(29 \%)$ had one or more mutations (total of 31 mutations in grandmothers) that differed from those in their grandchildren. Of these mutations, 29 were transitions $(17 \mathrm{C} \rightarrow \mathrm{T}, 8 \mathrm{~T} \rightarrow \mathrm{C}, 4 \mathrm{~A} \rightarrow \mathrm{G})$ and two were transversions $(\mathrm{T} \rightarrow \mathrm{G}$ and $\mathrm{A} \rightarrow \mathrm{T}$ ).

b) Of the three families $(21,27$ and 30$)$ in which an indication of homoplasmic mutations was detected in grandmothers in the first phase of the study, only in the grandmother from family 27 was the homoplasmic status confirmed for mutation 16,111 CT. Despite its absence in her grandchildren, this mutation was probably present in the mitochondrial lineage by inheritance since all clones shared the same mutation. The absence of this mutation in the two descendants suggests its loss probably in the mitochondrial lineage of generation 2. This grandmother was also heteroplasmic for three other novel mutations $(16,062$ AG, 16,109 AT and 16,351 AG) indicative of somatic alterations in the family. The grandmother of family 21 who was homoplasmic for the $16,188 \mathrm{CT}$ mutation in the first phase of the study showed this condition in only $10 \%$ of the clones. In addition, she had three novel mutations at positions $16,187 \mathrm{TC}, 16,294 \mathrm{TC}$ and $16,321 \mathrm{CT}$. In the grandmother of family 30 , mutation $16,131 \mathrm{TC}$ was present in only $30 \%$ of the clones.

c) Cloning and sequencing confirmed the heteroplasmic state in six $(1,3,9,11,13$, and 19) of the nine families in which the grandmothers were initially positive for this condition, with the mutations being present in $10 \%-40 \%$ of the clones analyzed. In the other three families $(5,8$ and 15$)$, a single mitochondrial lineage was present in the ten clones analyzed from each of them.

d) In the 120 clones investigated, there was an average of 0.1333 point mutation/422 bp sequence and an average of 0.0003159 mutation/nucleotide (corresponding to 352 mutations per million base pairs), with mutation frequencies varying from 237 to $3791 / 10^{6}$ bp (Table 1).

Various studies in the last two decades have examined the intricate mechanisms that govern aging (Richter et al., 1988; Ortopassi and Arnheim, 1990; Attardi, 2002; Mandavilli et al., 2002; Rose et al., 2002; Liang and Godley, 2003). However, to the best of our knowledge, most studies done so far have not used comparisons between family generations to assess the effects of aging on mutations in mtDNA. In this work, we investigated the influence of aging on the occurrence of somatic mutations in mtDNA by comparing mitochondrial lineages from grandmothers (elderly women) and their daughters' offspring. Analysis of the D-loop region of mtDNA revealed that 29\% $(9 / 31)$ of the grandmothers had point mutations that were absent in their grandchildren, and $26 \%$ were heteroplasmic, with two or more mitochondrial lineages. It is possible that both of these situations were a long-term consequence of mitochondrial oxidative phosphorylation that can lead to the accumulation of mtDNA mutations throughout life. In addition, the occurrence of these mutations may have been
Table 1 - Frequency of mtDNA point mutations in elderly women investigated by DNA cloning and sequencing (422 bp insert; 10 clones per individual).

\begin{tabular}{lcc}
\hline Sample & $\begin{array}{c}\text { Mutations (substitutions) } \\
\text { per individual }\end{array}$ & $\begin{array}{c}\text { Frequency of mutations per } \\
10^{6} \text { nucleotides }\end{array}$ \\
\hline $1 \mathrm{~A}$ & 1 & 237 \\
$3 \mathrm{~A}$ & 2 & 474 \\
$5 \mathrm{~A}$ & 0 & - \\
$8 \mathrm{~A}$ & 0 & - \\
$9 \mathrm{~A}$ & 2 & 948 \\
$11 \mathrm{~A}$ & 4 & 1896 \\
$13 \mathrm{~A}$ & 1 & 237 \\
$15 \mathrm{~A}$ & 0 & - \\
$19 \mathrm{~A}$ & 1 & 237 \\
$21 \mathrm{~A}$ & 4 & 3791 \\
$27 \mathrm{~A}$ & 3 & 2133 \\
$30 \mathrm{~A}$ & 3 & 711 \\
\hline
\end{tabular}

enhanced by the inefficiency of the mtDNA repair mechanisms in the presence of high levels of oxygen free radicals (Richter et al., 1988; Rose et al., 2002; Liang and Godley, 2003; Santos et al., 2005).

Most of the heteroplasmic grandmothers were $>60$ years old, although somatic mutations were also observed in two women who were 40 and 55 years old. Of the 90 clones from grandmothers with altered sequences, 27 had $=1$ mutation/clone. A 40-year-old grandmother (family 27) had a mutation at position 16,111 in all of the clones analyzed. She also had a mutation at position 16,062 in a single clone, and mutations at positions 16,109 and 16,351 in another clone. None of these four mutations was present in her grandchildren.

Previous studies have suggested a selective advantage in the expansion and maintenance of somatic mutations in different generations (Santos et al., 2005, 2008). He et al. (2010) demonstrated that healthy individuals are characterized by a complex mixture of related mitochondrial genotypes rather than a single genotype. Some studies have suggested that certain point mutations are more frequent in elderly subjects (Michikawa et al., 1999; Chinnery et al., 2001). In our sample, no specific mutation had a higher frequency than the others, but a large number of mutations were identified in the grandmothers that were not observed in their descendants. This result suggests that mtDNA studies done using unrelated individuals can yield misleading conclusions about the frequency of hotspot mutations in elderly subjects.

\section{Acknowledgments}

The authors thank the families who agreed to participate in this study. This work was supported by RENOR/CAPES (Rede Norte para Formação e Fixação de 
Grupos de Docentes Pesquisadores na Amazônia), Milênio/CNPq (Conselho Nacional de Desenvolvimento Científico e Tecnológico), FINEP (Financiadora de Estudos e Projetos) and UFPA (Universidade Federal do Pará).

\section{References}

Attardi G (2002) Role of mitochondrial DNA in human aging. Mitochondrion 2:27-37.

Anderson S, Bankier AT, Barrell BG, De Bruijn MH, Coulson AR, Drouin J, Eperon IC, Nierlich DP, Roe BA and Sanger F (1981) Sequence and organization of the human mitochondrial genome. Nature 290:457-465.

Andrews RM, Kubacka I, Chinnery PF, Lightowler RN, Turnbull DM and Howell N (1999) Reanalysis and revision of the Cambridge reference sequence for the human mitochondrial DNA. Nat Genet 23:147.

Ayres M, Ayres Jr M, Ayres DL and Santos AAS (2007) BioEstat: Aplicações Estatísticas nas Áreas das Ciências Biológicas e Médicas. 5th edition. Sociedade Civil Mamirauá, Belém, $363 \mathrm{pp}$.

Chinnery PF, Taylor GA, Howell N, Brown DT, Parsons TJ and Turnbull DM (2001) Point mutations of the mtDNA control region in normal and neurodegenerative human brains. Am J Hum Genet 68:529-532.

Corral-Debrinski M, Horton T, Lott MT, Shoffner JMM, Beal F and Wallace DC (1992) Mitochondrial DNA deletions in human brain: Regional variability and increase with advanced age. Nat Genet 2:324-329.

Harman D (1981) The aging process. Proc Natl Acad Sci USA 78:7124-7128.

He Y, Wu J, Dressman DC, Iacobuzio-Donahue C, Markowitz SD, Velculescu VE, Diaz Jr LA, Kinzler KW, Volgelstein B and Papadopoulos N (2010) Heteroplasmic mitocondrial DNA mutations in normal and tumor cells. Nature 464:610-616.

Khaidakov M, Heflich RH, Manjanatha MG, Myers MB and Aidoo A (2003) Accumulation of point mutations in mitochondrial DNA of aging mice. Mutat Res 526:1-7.

Liang F and Godley BF (2003) Oxidative stress-induced mitochondrial DNA damage in human retinal pigment epithelial cells: A possible mechanism for RPE aging and age-related macular degeneration. Science 76:1-7.

Miquel J (1998) An update on the oxygen stress-mitochondrial mutation theory of aging: Genetic and evolutionary implications. Exp Gerontol 33:113-126.

Mandavilli BS, Santos JH and Van Houten B (2002) Mitochondrial DNA repair and aging. Mutat Res 509:127-151.
Michikawa Y, Mazzucchelli F, Bresolin N, Scarlato G and Attardi G (1999) Aging-dependent large accumulation of point mutations in the human mtDNA control region for replication. Science 286:774-779.

Ortopassi GA and Arnheim N (1990) Detection of a specific mitochondrial DNA deletion in tissues of older humans. Nucleic Acids Res 18:6927-6933.

Parsons TJ, Muniec DS, Sullivan K, Woodyatt N, Alliston-Greiner R, Wilson MR, Berry DL, Holland KA, Weedn VW, Gill $\mathrm{P}$, et al. (1997) A high observed substitution rate in the human mitochondrial DNA control region. Nat Genet 15:363368.

Richter C, Park JW and Ames BN (1988) Normal oxidative damage to mitochondrial and nuclear DNA. Proc Natl Acad Sci USA 85:6465-6467.

Rose G, Passarino G, Franceschi C and De Benedictis G (2002) The variability of the mitochondrial genome in human aging: A key for life and death? Int J Biochem Cell Biol 34:1449-1460.

Sambrook J, Fritsch EF and Maniatis T (1989) Molecular Cloning: A Laboratory Manual. Cold Spring Harbor Press, Cold Spring Harbor.

Santos C, Montiel R, Arruda A, Alvarez L, Aluja MP and Lima M (2008) Mutation patterns of mtDNA: Empirical inferences for the coding region. BMC Evol Biol 8:167.

Santos C, Montiel R, Sierra B, Bettencourt C, Fernandez E, Alvarez L, Lima M, Abade A and Aluja MP (2005) Understanding differences between phylogenetic and pedigreederived mtDNA mutation rate: A model using families from the Azores Islands (Portugal). Mol Biol Evol 22:1490-1505.

Wallace DC (1992) Mitochondrial genetics: A paradigm for aging and degenerative diseases? Science 256:628-632.

\section{Supplementary Material}

The following online material is available for this article:

Table S1 - Mutations identified by direct sequencing of the mtDNA D-loop region in 93 individuals (generations F1 and F3).

Table S2 - Mutations observed in the mtDNA of 120 clones from 12 grandmothers.

This material is available as part of the online article from http://www.scielo.br/gmb.

$$
\text { Editor: Angela M. Vianna-Morgante }
$$

License information: This is an open-access article distributed under the terms of the Creative Commons Attribution License, which permits unrestricted use, distribution, and reproduction in any medium, provided the original work is properly cited. 
.Table S1: Mutations identified by direct sequencing of the mtDNA D-loop region in 93 individuals (generations F1 and F3).

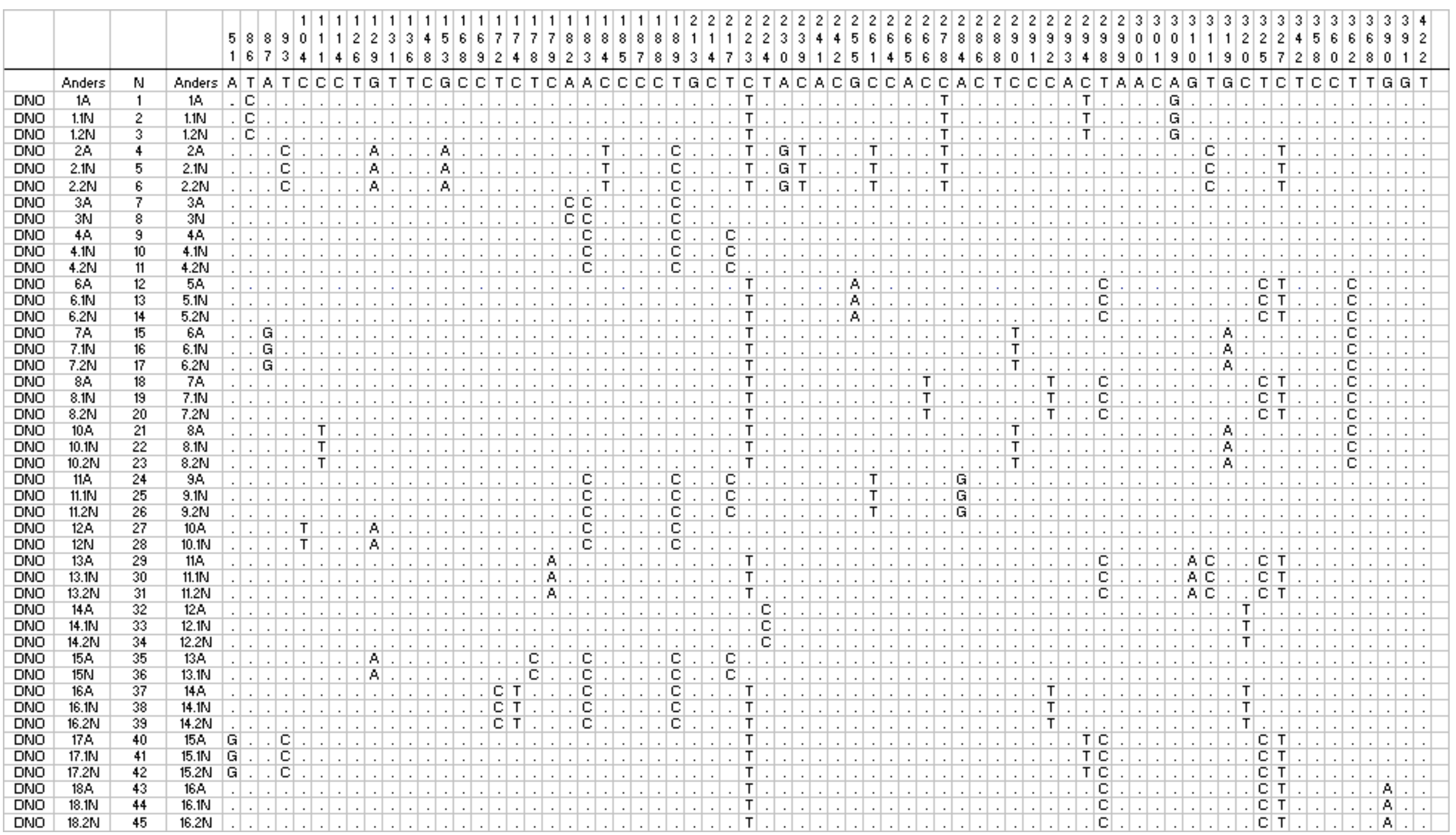




\section{Continuation:}

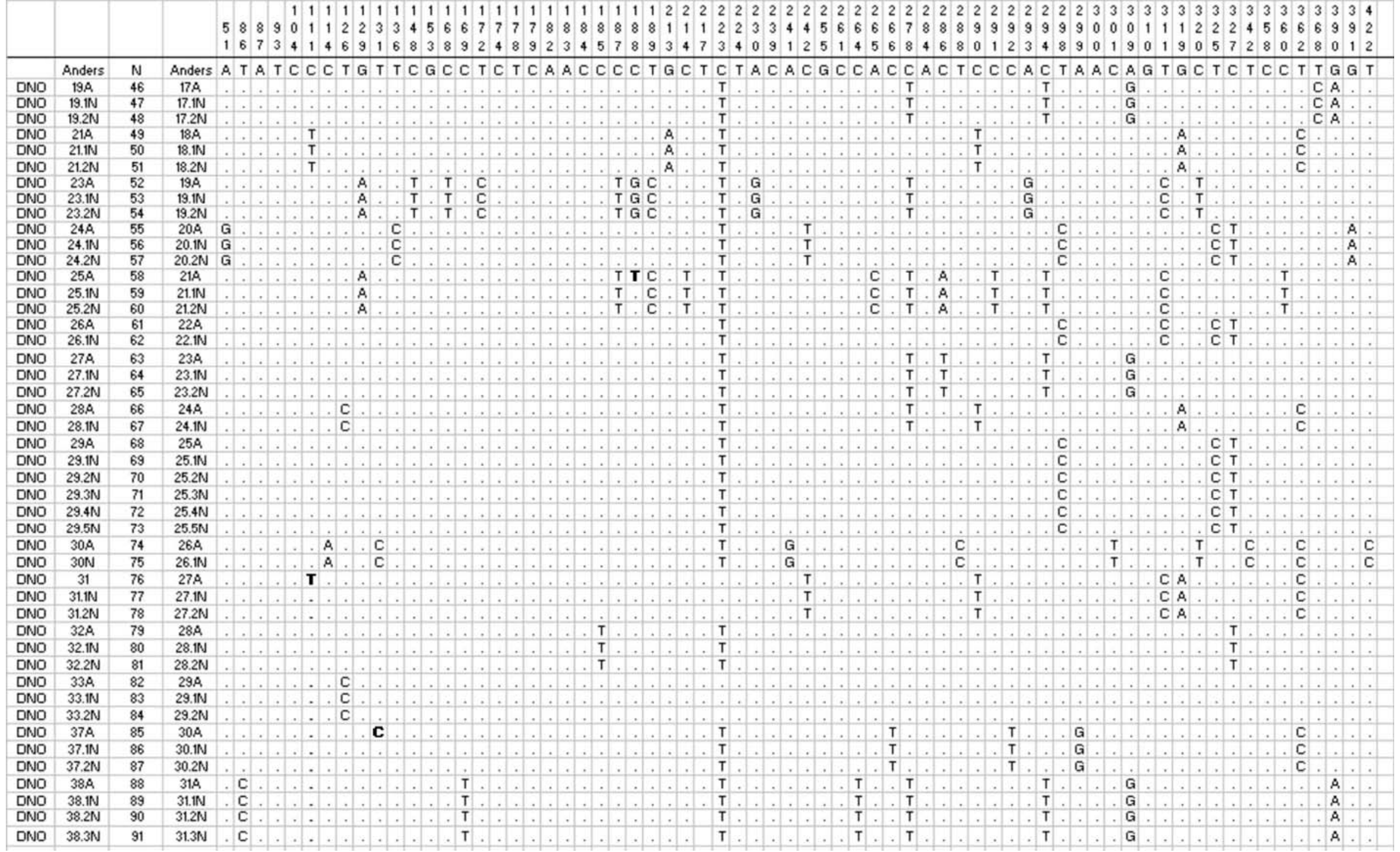

A - elderly individuals (F1); $\mathrm{N}$ - their young controls (F3); the different types of mutations are indicated in the table in bold. 
Table S2: Mutations observed in the mtDNA of 120 clones from 12 grandmothers.

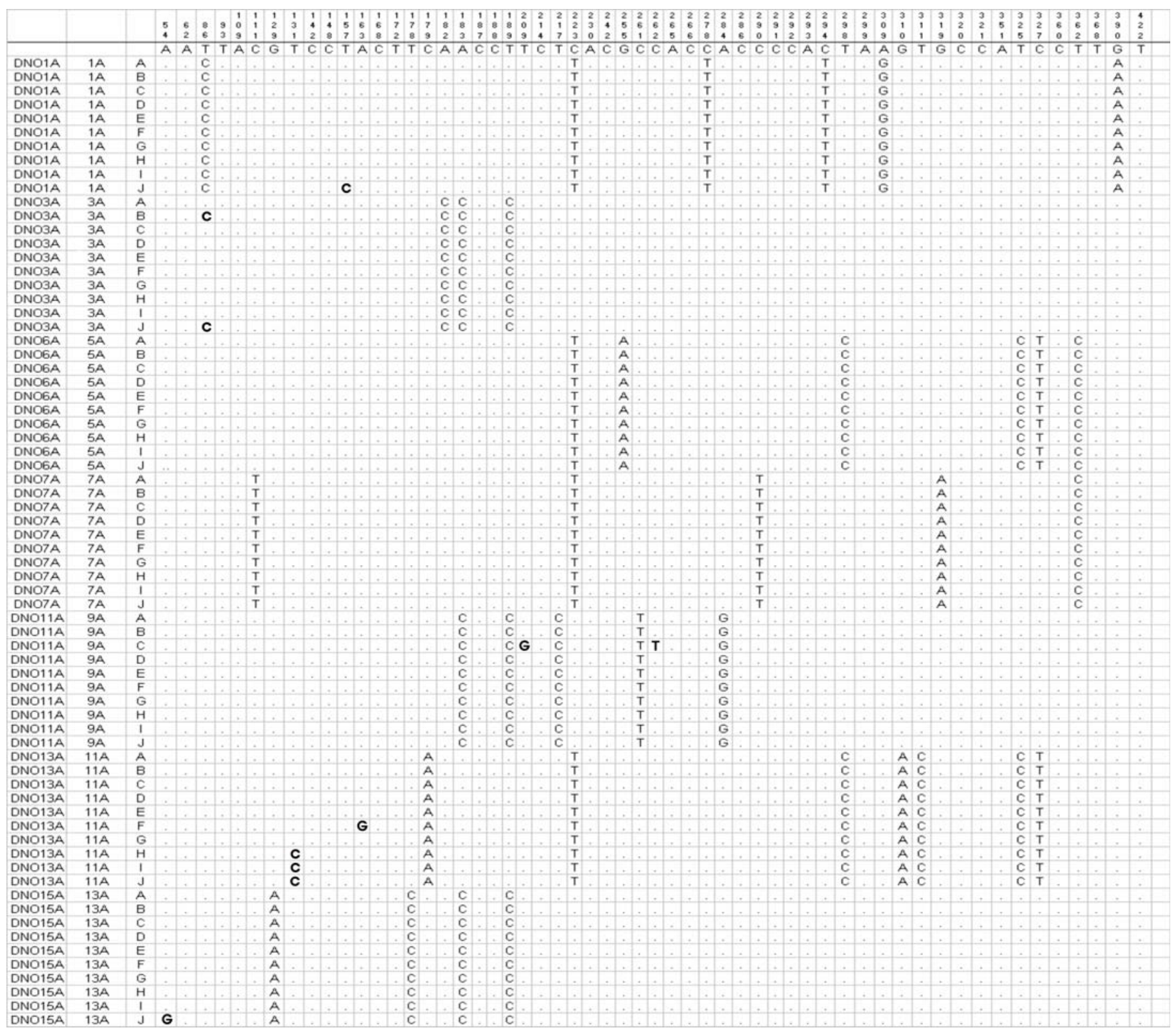




\section{Continuation}

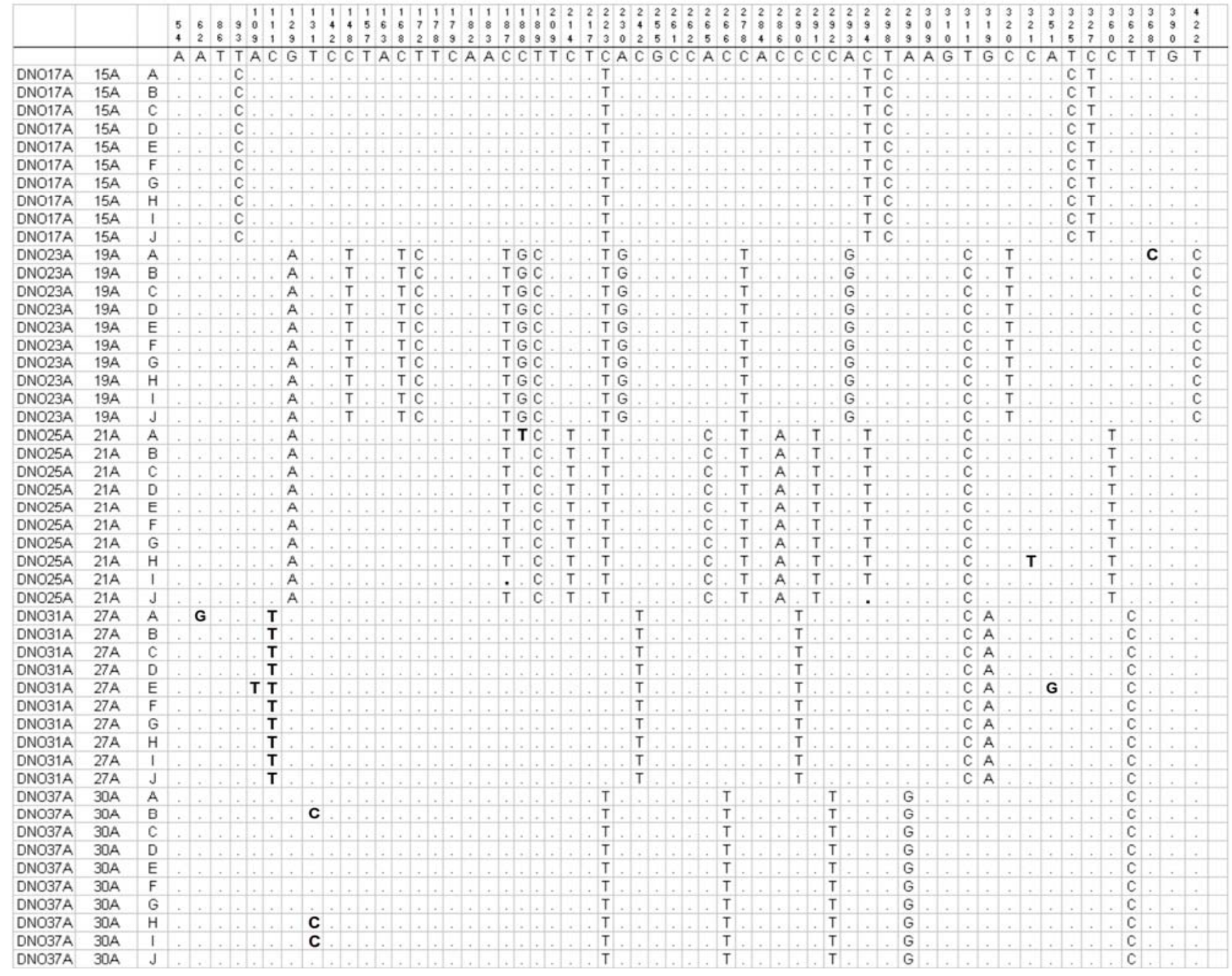

The different types of mutations are indicated in the table in bold. 